\title{
J- AND H-BAND OBSERVATIONS OF THE GALACTIC BULGE WITH PANIC
}

\author{
SHIGERU MATSUMOTO \\ Department of Astronomy, University of Tokyo \\ Bunkyo-ku,Tokyo,113-0033,Japan \\ YOSHIKAZU NAKADA \\ Kiso Observatory,Institute of Astronomy, University of Tokyo \\ Mitake-mura,Nagano,397-0101,Japan
}

AND

IAN S. GLASS

South African Astronomical Observatory

PO Box 9, Observatory 7935, South Africa

\section{Observations}

Nine regions in the Galactic bulge were observed at $\mathrm{J}$ and $\mathrm{H}$ bands with a PtSi $1040 \times 520$ array camera which is named the PtSi Astronomical Near Infrared Camera (PANIC). These regions were centered at $1=\left(-5^{\circ}, 0^{\circ},+5^{\circ}\right)$ $\mathrm{b}=\left(-6^{\circ}, 0^{\circ},+6^{\circ}\right)$ respectively. Each region was covered with nine frames each of which spanned $30^{\prime}$ by $30^{\prime}$ square arcmin. Observations were carried out at the South African Astronomical Observatory, Cape Town, from 1995 to 1997 using a $40 \mathrm{~cm} \mathrm{f} / 5$ Newtonian telescope. A bolometric correction (Frogel and Whitford 1987) was given to the observed stars by using $\mathrm{J}-\mathrm{H}$ values of reference RGB stars (Frogel et al 1990).

\section{Profiles}

The observed stars were grouped into three magnitude ranges: A) $-4.0 \leq$ $\left.\mathrm{M}_{b o l} \leq-3.5, \mathrm{~B}\right)-3.5 \leq \mathrm{M}_{b o l} \leq-3.0$, and C) $-3.0 \leq \mathrm{M}_{b o l} \leq-2.5$. For each bolometric magnitude range the number densities were derived at 15 arcminutes intervals along the surveyed regions. In figure 1 the range A densities were scaled up by factor 1.61 while the range $C$ densities were scaled down by 

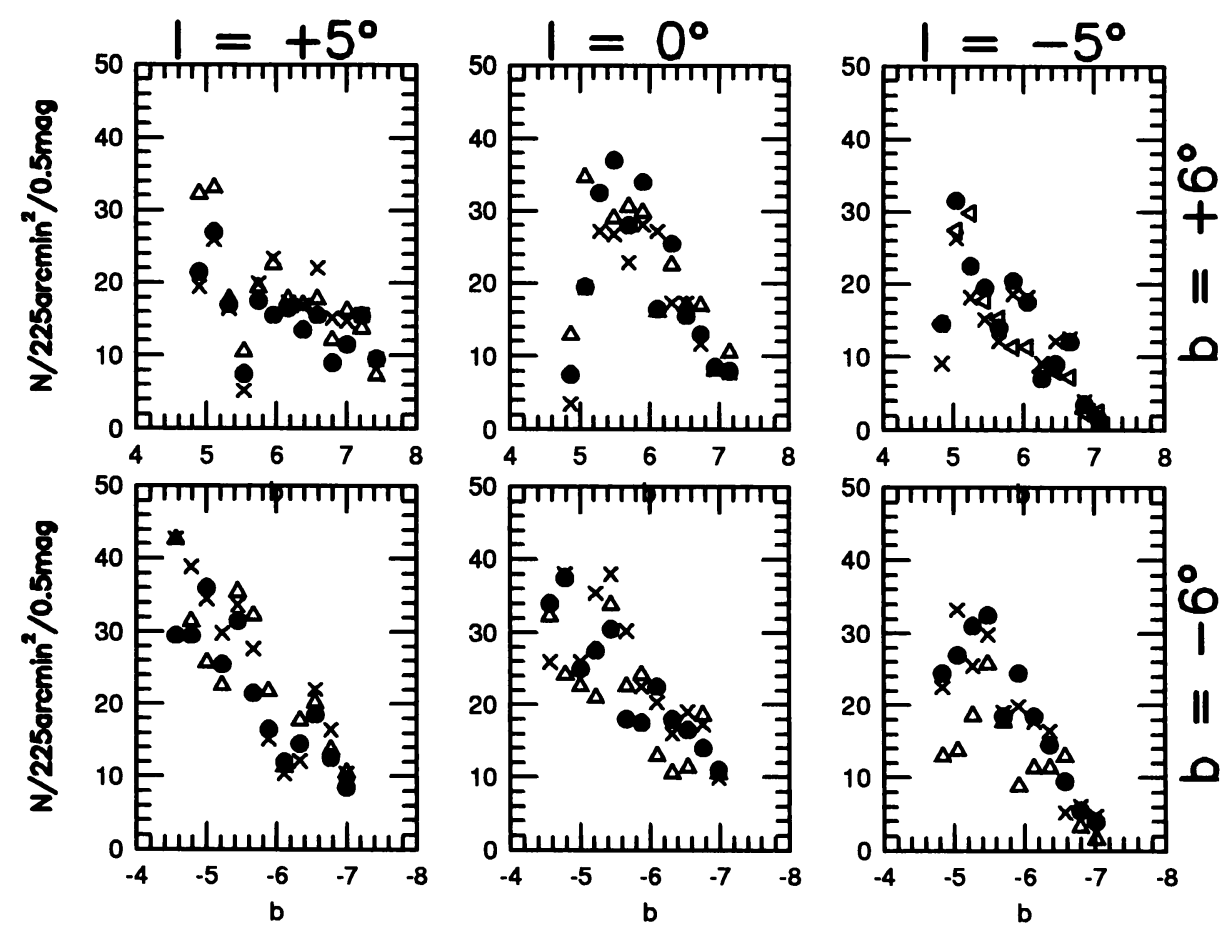

Figure 1. The Galactic Bulge Density Profiles. Symbols are $\Delta$ for $-4.0 \leq \mathrm{M}_{b o l} \leq-3.5$, - for $-3.5 \leq \mathrm{M}_{b o l} \leq-3.0, \times$ for $-3.0 \leq \mathrm{M}_{b o l} \leq-2.5$.

factor 0.86 in order to achieve a good overlap with the range $\mathrm{B}$ densities. It is easily seen that they are distributed in a narrow zone indicating that stars with different luminosities are well mixed throughout the bulge.

The most conspicuous feature in figure 1 is a rather flat density distribution of number density in the area $l=5^{\circ}, b=6^{\circ}$. This feature is expected when the elongated bulge inclines toward the eastern side. Also the density difference between the east $\left(l=5^{\circ}\right)$ and the west $\left(l=-5^{\circ}\right)$ side can be caused by the slanted direction of the bulge. Detailed examination of the number distribution is under way to determine the shape of the Galactic bulge from the number count of the infrared sources.

\section{References}

Frogel J.A., Whitford A.E. (1987) Ap.J., 320, 199-237

Frogel J.A., Terndrup D.M., Blanco V.M., Whitford A.E. (1990) Ap.J., 353, 494-523 